\title{
A Comparison of Three Different Methods for Classification of Breast Cancer Data
}

\author{
Daniele Soria Jonathan M. Garibaldi \\ University of Nottingham \\ School of Computer Science \\ Jubilee Campus, Wollaton Road, \\ Nottingham, NG8 1BB, UK \\ \{dqs,jmg\}@cs.nott.ac.uk
}

\author{
Elia Biganzoli \\ National Cancer Institute of Milan \\ Unit of Medical Statistics and Biometry \\ Via Vanzetti 5, 20133 Milan, Italy \\ elia.biganzoli@unimi.it
}

\author{
Ian O. Ellis \\ University of Nottingham \\ School of Molecular Medical Sciences \\ Queens Medical Centre, Derby Road, \\ Nottingham, NG7 2UH, UK \\ ian.ellis@nottingham.ac.uk
}

\begin{abstract}
The classification of breast cancer patients is of great importance in cancer diagnosis. During the last few years, many algorithms have been proposed for this task. In this paper, we review different supervised machine learning techniques for classification of a novel dataset and perform a methodological comparison of these. We used the C4.5 tree classifier, a Multilayer Perceptron and a naive Bayes classifier over a large set of tumour markers. We found good performance of the Multilayer Perceptron even when we reduced the number of features to be classified. We found naive Bayes achieved a competitive performance even though the assumption of normality of the data is strongly violated.
\end{abstract}

\section{Introduction}

Worldwide, breast cancer is the second most common type of cancer and the fifth most common cause of cancer death. This disease poses a serious threat for women's health. Since the early years of cancer research, biologists have used the traditional microscopic technique to assess tumour behavior for breast cancer patients. Precise prediction of tumours is critically important for the diagnosis and treatment of cancer. Modern machine learning techniques are progressively being used by biologists to obtain proper tumour information from the databases. Among the existing techniques, supervised learning methods are the most popular in cancer diagnosis [8].

According to John and Langley [6], methods for inducing probabilistic descriptions from training data have emerged as a major alternative to more established approaches to machine learning, such as decision-tree induction and neural networks. However, some of the most impressive results to date have come from a much simpler and much older - approach to probabilistic induction known as the naive Bayesian classifier. Despite the simplifying assumptions that underlie the naive Bayesian classifier, experiments on real-world data have repeatedly shown it to be competitive with much more sophisticated induction algorithms. Furthermore, naive Bayes can deal with a large number of variables and large data sets, and it handles both discrete and continuous attribute variables.

In this paper, we present a comparison of three different classifiers that may be used in machine learning, namely the naive Bayes algorithm, the $\mathrm{C} 4.5$ decision tree and the Multilayer Perceptron function. The same machine learning techniques were already used in literature: in particular, Bellaachia and Guven in [1], revising a study of Delen et al. [3], used the above methods to find the most suitable one for predicting survivability rate of breast cancer patients. Our study was instead motivated by the necessity to find an automated and robust method to validate our previous classification of breast cancer markers [4]. We had, in fact, obtained six classes using agreement between different clus- 


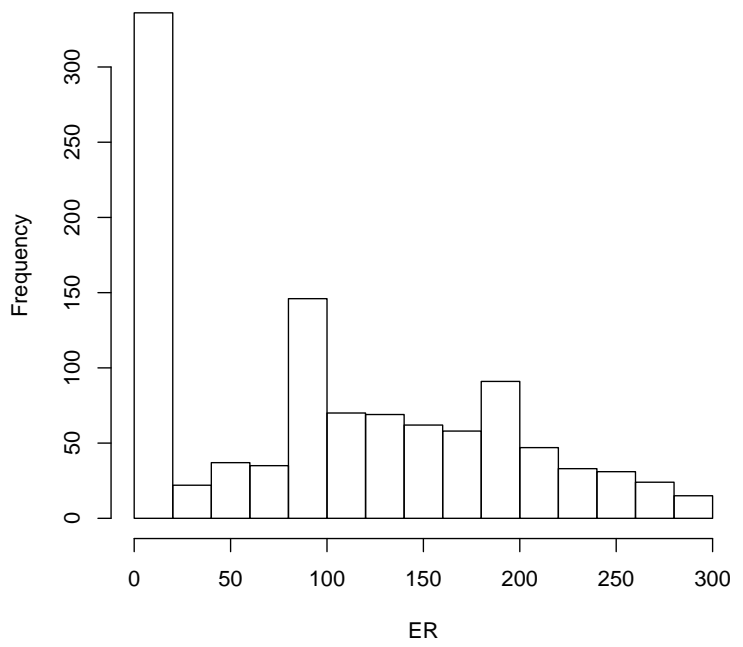

Figure 1. Histogram of variable ER

tering algorithms. Starting from these groups, we wanted to reproduce the classification keeping into account the high non-normality of our data (see Figure 1 and 2). For this reason we started using the $\mathrm{C} 4.5$ and the Multilayer Perceptron classifiers and then we compare results with the naive Bayes one. Surprisingly, we found that when we reduce our dataset to ten markers, the naive Bayes classifier performs better than the $\mathrm{C} 4.5$, even though the normality assumption is strongly violated by our features' distribution.

A Bayesian classifier is a fast-supervised classification technique which is suitable for large-scale prediction and classification tasks on complex and incomplete datasets. Naive Bayesian classification performs well if the values of the attributes for the sessions are independent. The naive Bayes classifier applies to learning tasks where each instance $x$ is described by a conjunction of attribute values and where the target function $f(x)$ can take on any value from same finite set $V$ [7].

C4.5 builds decision trees from a set of training data, using the concept of Information Entropy. Each attribute of the data can be used to make a decision that splits the data into smaller subsets. C4.5 examines the normalized information gain (difference in entropy) that results from choosing an attribute for splitting the data. The attribute with the highest normalized information gain is the one used to make the decision. The algorithm then recurs on the smaller sublists.

A Multilayer Perceptron is a feed-forward network with one or more layers of nodes between the input and output layers of nodes. These additional layers contain hidden

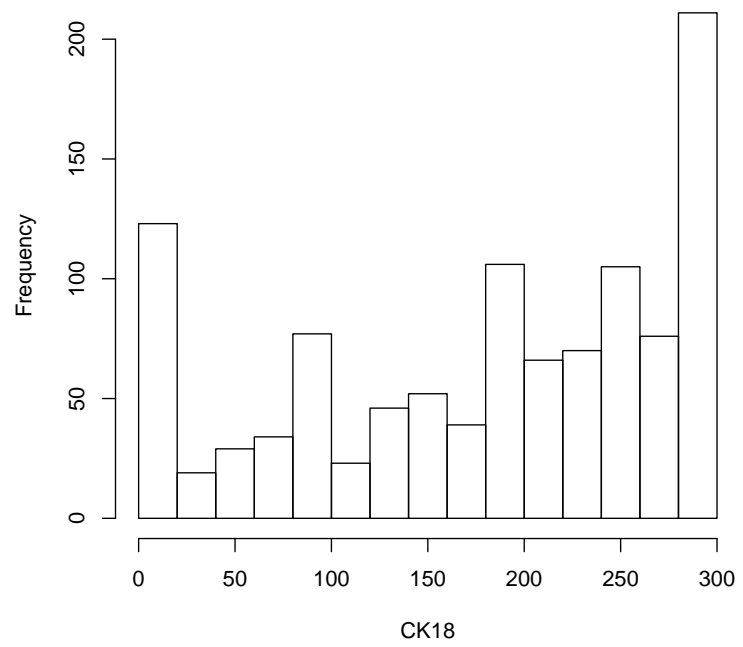

Figure 2. Histogram of variable CK18

nodes that are not directly connected to both the input and the output nodes. The capabilities of the Multilayer Perceptrons come from the non-linearity used in these nodes. The number of nodes in the hidden layer must be large enough to form a decision region that is as complex as required by a given problem.

The paper is organized as follows: in Section 2, a description of each technique is reported. Then in Section 3 we introduce the dataset worked on and explain in detail the results obtained from the three classifiers using the WEKA software [11]. Section 4 is reserved for discussion and future work.

\section{Methods}

\subsection{C4.5 Classifier}

C4.5 is an algorithm used to generate a decision tree developed by Ross Quinlan. C4.5 is an extension of Quinlan's earlier ID3 algorithm. The decision trees generated by $\mathrm{C} 4.5$ can be used for classification, and for this reason, C4.5 is often referred to as a statistical classifier.

Tree induction methods are considered to be supervised classification methods, which generate decision trees derived from a particular data set. C4.5 uses the concept of information gain to make a tree of classificatory decisions with respect to a previously chosen target classification [9]. The output of the system is available as a symbolic rule base. The cases, described by any mixture of nominal and numeric properties, are scrutinized for patterns that 
allow the classes to be reliably discriminated. These patterns are then expressed as models, in the form of decision trees or sets of if-then rules, which can be used to classify new cases, with an emphasis on making the models understandable as well as accurate [9]. For real world databases the decision trees become huge and are always difficult to understand and interpret. In general, it is often possible to prune a decision tree to obtain a simpler and more accurate tree [9].

\subsection{Multilayer Perceptron Classifier}

A Multilayer Perceptron is a feedforward artificial neural network model that maps sets of input data onto a set of appropriate output. It is a modification of the standard linear perceptron in that it uses three or more layers of neurons (nodes) with nonlinear activation functions, and is more powerful than the perceptron in that it can distinguish data that is not linearly separable, or separable by a hyperplane [5].

Multilayer feedforward networks are an important class of neural networks. Typically, the network consists of a set of sensory units that constitute the input layer, one or more hidden layers of computation nodes, and an output layer of computation nodes. Multilayer Perceptrons have been applied successfully to solve some difficult and diverse problems by training them in a supervised manner with a highly popular algorithm known as the error back-propagation algorithm. Basically, error back propagation learning consists of two passes through the different layers of the network: a forward pass and a backward pass. In the forward pass, an activity pattern (input vector) is applied to the sensory nodes of the network, and its effect propagates through the network layer by layer. Finally, a set of outputs is produced as the actual response of the network. During the forward pass the synaptic weights of the network are all fixed. During the backward pass, on the other hand, the synaptic weights are all adjusted in accordance with an error-correction rule. Specifically, the actual response of the network is subtracted from a desired (target) response to produce an error signal. This error signal is the propagated backward through the network, against the direction of synaptic connections - hence the name "error back-propagation". The synaptic weights are adjusted to make the actual response of the network move closer to the desired response in a statistical sense.

A Multilayer Perceptron has three distinctive characteristics:

1. The model of each neuron in the network includes a nonlinear activation function.

2. The network contains one or more layers of hidden neurons that are not part of the input or output of the network. These hidden neurons enable the network to learn complex tasks by extracting progressively more meaningful features from the input patterns (vectors).

3. The network exhibits a high degree of connectivity, determined by the synapses of the network. A change in the connectivity of the network requires a change in the population of synaptic connections or their weights.

It is through the combination of these characteristics together with the ability to learn from experience through training that the Multilayer Perceptron derives its computing power [5].

\subsection{Naive Bayes Classifier}

A naive Bayes classifier is a simple probabilistic classifier based on applying Bayes' theorem with strong independence assumptions. The performance goal is to predict the class of test instances as accurately as possible. This kind of classifier is termed naive because it is based on two simplifying common assumptions: firstly, it assumes that the predictive attributes are conditionally independent given the class and secondly, the values of numeric attributes are normally distributed within each class.

Naive Bayes treats discrete and continuous attributes somewhat differently. For each discrete attribute, the probability that the attribute $X$ will take on the particular $x$ when the class is $c$ is modeled by a single real number between 0 and 1. In contrast, each continuos attribute is modeled by some continuous probability distribution over a range of that attribute's values.

Let $C$ be the random variable denoting the class of an instance and $X$ be a vector of random variables denoting the observed attribute values. Let $c$ be a particular class label and $x$ represent a particular observed attribute value. If we have a test case $x$ to classify, the probability of each class given the vector of observed values for the predictive attributes may be obtained using the Bayes' theorem:

$$
p(C=c \mid X=x)=\frac{p(C=c) p(X=x \mid C=c)}{p(X=x)}
$$

and then predicting the most probable class. Because the event is a conjunction of attribute values assignments, and because of the attributes conditional independence assumption, the following equation may be written:

$$
p(X=x \mid C=c)=\prod_{i} p\left(X_{i}=x_{i} \mid C=c\right) .
$$

which is quite simple to calculate for training and test data [6]. 


\section{Experiments and Results}

\subsection{Patients}

A series of 1076 patients from the Nottingham Tenovus Primary Breast Carcinoma Series were used in a previous study [4] where we had applied different clustering techniques to classify data in robust and clinically significant groups. For clustering analyses, we had used a panel of 25 tumour markers, which are listed in Table 1. Consensus between four different clustering techniques was used to determine six core classes. $62 \%$ (663 cases) of the population was classified into one of these six groups, while the remaining $38 \%$ presented indeterminate or mixed characteristics. In this study we only focused on the subset of the 'in-class' cases to run the classifiers on in order to find an automated way to justify and reproduce the classification obtained before [4]. This subset represents a novel clinical categorisation of breast cancer which is interesting in its own right and presents a challenging classification task. Further understanding of undetermined cases is left open for future investigation.

Still based on previous research [4], we selected 14 'important' markers candidates that were discriminant in the categorisation process and whose distribution was very different among the six classes. These 14 markers were selected on the basis of clinical importance as indicated by pathologists involved in previous studies. We then performed an exhaustive search of the best combination of 10 markers out of these 14 based on the naive Bayes classification results. This was done as reducing the number of markers used for classification is a clinical aim, as this would both simplify and reduce the costs of a clinical test based on these markers. We used this 'new' smaller dataset to repeat our experiments applying the above classifiers on this.

\subsection{Results}

After loading our data in the WEKA software [11], we chose the $\mathrm{C} 4.5$ algorithm classifier. As it can handle continuous attributes, there was no need to discretize any of the attributes and in our experiments we accepted the default values for the parameters. The default version does perform some pruning (using the subtree raising approach), but does not perform error pruning. We chose to run the classifier 10 times using the 10-fold cross validation option and evaluate the accuracy of the obtained classification simply by looking at the percentage of the corrected classify instances. We will use the same 'initial conditions' and we will repeat the experiments for the same number of times also when running the other classifiers. We will then compute the mean of the returning results. The results we obtained were quite good, precisely we got 582 cases correctly

\begin{tabular}{ll} 
Antibody, clone & Short Name \\
\hline Luminal phenotype & \\
CK 7/8 [clone CAM 5.2] & CK7/8 \\
CK 18 [clone DC10] & CK18 \\
CK 19 [clone BCK 108] & CK19 \\
Basal Phenotype & \\
CK 5/6 [cloneD5/16134] & CK5/6 \\
CK 14 [clone LL002] & CK14 \\
SMA [clone 1A4] & Actin \\
p63 ab-1 [clone 4A4] & p63 \\
Hormone receptors & ER \\
ER [clone 1D5] & PgR \\
PgR [clone PgR 636] & AR \\
AR [clone F39.4.1] & \\
EGFR family members & EGFR \\
EGFR [clone EGFR.113] & HER2 \\
HER2/c-erbB-2 & HER3 \\
HER3/c-erbB-3 [clone RTJ1] & HER4 \\
HER4/c-erbB-4 [clone HFR1] & \\
Tumour suppressor genes & p53 \\
p53 [clone DO7] & nBRCA1 \\
nBRCA1 Ab-1 [clone MS110] & FHIT \\
Anti-FHIT [clone ZR44] & \\
Cell adhesion molecules & E-cad \\
Anti E-cad [clone HECD-1] & P-cad \\
Anti P-cad [clone 56] & \\
Mucins & MUC1 \\
NCL-Muc-1 [clone Ma695] & MUC1co \\
NCL-Muc-1 core [clone Ma552] & MUC2 \\
NCL muc2 [clone Ccp58] & \\
Apocrine differentiation & GCDFP \\
Anti-GCDFP-15 & \\
Neuroendocrine differentiation & Shromo \\
Chromogranin A [clone DAK-A3] & \\
Synaptophysin [clone SY38] & Synapto \\
\hline
\end{tabular}

Table 1. Antibodies used and their dilutions

classified (87.8\%) and just 81 (12.2\%) incorrectly classified. Our main concern in using this classifier came from the set of rules that were produced: they appear to be quite numerous and not straightforward, especially if they should be used by scientists not familiar with computational analysis.

We then considered the Multilayer Perceptron classifier: again we used the default parameters, leaving the number of neurons in the hidden layer as 15 , which is the sum of the number of attributes and classes divided by two. The default backpropagation learning algorithm was used. Compari- 
son of alternative learning algorithms is outside the scope of this study. This method performed better than the C4.5 succeeding in correctly classifying 647 instances $(97.6 \%)$ out of 663 ; just 16 cases $(2.4 \%)$ were misclassified.

We finally applied the naive Bayes classifier, which is based on the assumption that numeric attributes are conditionally independent. This method performed worse than the previous ones, classifying properly a smaller amount of cases (576, corresponding to $86.9 \%$ ). A summary of the above results can be found in Table 2 .

\section{Whole data}

\begin{tabular}{lcc} 
Method & Classified & Misclassified \\
\hline C4.5 & $582(87.8 \%)$ & $81(12.2 \%)$ \\
\hline MLP & $647(97.6 \%)$ & $16(2.4 \%)$ \\
\hline NB & $576(86.9 \%)$ & $87(13.1 \%)$ \\
\hline
\end{tabular}

Table 2. Comparison of results on three classifiers using 25 markers.

As we previously reported we considered a smaller dataset containing just 10 'important' markers. We repeated our experiments applying the above classifiers on this 'new' smaller dataset. For the $\mathrm{C} 4.5$ decision tree we could not see a particular difference, having 581 cases $(87.6 \%)$ correctly classified. Also for the Multilayer Perceptron (MLP) we have an increased number of misclassified instances, this time being $34(5.1 \%)$. The naive Bayes (NB), instead, performed very well compared to the previous run. Now we found that 617 cases $(93.1 \%)$ were classified properly and just $46(6.9 \%)$ were misclassified.

A summary of the latter results is reported in Table 3.

\begin{tabular}{lcc}
\multicolumn{2}{c}{ Ten Markers } \\
Method & Classified & Misclassified \\
\hline C4.5 & $581(87.6 \%)$ & $82(12.4 \%)$ \\
\hline MLP & $629(94.9 \%)$ & $34(5.1 \%)$ \\
\hline NB & $617(93.1 \%)$ & $46(6.9 \%)$ \\
\hline
\end{tabular}

Table 3. Comparison of results on three classifiers using only 10 markers.

As Bouckaert did in [2], the 10 accuracies of each algorithm were compared using t-tests, after checking for normality using the Shapiro test [10]. We found that, for both the whole data and the 10-markers datasets, the Multilayer Perceptron classifier performed significantly better than the other two $(p<<0.01)$. The C4.5 decision tree algorithm was significantly more accurate than the naive Bayes $(p<0.01)$ when we consider the whole data, but was not when we reduced the number of features. Table 4 summarizes our findings.

\begin{tabular}{cccc}
\multicolumn{4}{c}{ Average accuracies } \\
& $C 4.5$ & MLP & $N B$ \\
\hline Whole data & $87.8(6.3)$ & $97.6(1.8)$ & $86.9(2.5)$ \\
10 Markers & $87.6(6.6)$ & $94.9(2.6)$ & $93.1(2.5)$ \\
\hline
\end{tabular}

Table 4. Average accuracies on $10 \times 10$ cross validation experiments for the three classifiers (standard deviation in brackets).

\section{Discussion}

In this paper we reviewed three different classifiers and used them over a novel dataset of tumour markers for breast cancer. From our experiments we got different results for each of them.

Using the whole dataset $(25$ markers $\times 663$ instances) we obtained the best performance from the Multilayer Perceptron classifier: in fact just 16 cases were incorrectly classified. The naive Bayes and C4.5 decision tree returned similar results (but worse than the MLP), with the latter being a bit more accurate than the naive Bayes.

When we moved to consider just the 10 'most important' markers, we found a substantial improvement in the naive Bayes performance: even though it did not return the highest number of correctly classified instances, it performed much better than with all the markers, decreasing the number of misclassified instances from 87 to 46. Again, the best results were obtained using the Multilayer Perceptron, but this time the network did not perform as well as before: there were 18 more cases of misclassification. Finally, the C4.5 decision tree was the worst classifier among the three used, performing almost identically as with all markers.

From the results, all classifiers achieved a reasonable performance. They all are suitable for large-scale prediction and classification tasks on complex datasets. However, each of them has weak points.

The C4.5 classifier may be considered what is called 'a white box model': the reason for arriving at the classification can be explicitly determined by examining the model. It also achieves good classification accuracy with large data in a short time.

On the other hand, for real world datasets, the decision tree may become huge. In particular, for scientists not fa- 
miliar with computational analysis, the set of rules coming from a decision tree may not be straightforward.

Multilayer Perceptrons, using a backpropagation algorithm, are a standard algorithm for any supervised-learning pattern recognition process. However, like the majority of neural networks, it is a good example of a 'black box model', since explanation of the results is not available in an easily comprehended form. If one tries to write down the network model and the function representing the entire process, this might take a long time and in some cases it might be extremely complicated.

Naive Bayes is a fast-supervised classification technique and, in general, it is a good approach for a classification problem. It is easy to understand and reproduce manually, being basically based on a product of conditional probabilities.

However, one must be aware that naive Bayes relies on two fundamental assumptions: the first one is the complete independence of features (which is largely satisfied in our data), and the second is that the attributes should follow a normal distribution, which is not always true. Considering the latter assumption, it is immediately apparent that our data does not have a normal distribution. However, despite the violation in its assumptions, the naive Bayesian classifier is remarkably effective on our dataset in practice, showing a good performance.

Given the violation of the naive Bayes hypothesis of normality, we have begun exploring other methods to represent features' distributions and to classify our data. We are now trying to implement a 'non-parametric' version of the naive Bayes classifier, which should be able to categorize instances independently from their distribution. In conclusion, we have found a standard MLP to be a highly effective classifier, but with poor interpretability. We have also found that naive Bayes achieves almost as good performance, with good interpretability, despite the strong violation of one of its assumptions.

\section{Acknowledgements}

This study was supported by the BIOPATTERN FP6 Network of Excellence (FP6-IST-508803) and the BIOPTRAIN FP6 Marie-Curie EST Fellowship (FP6-007597).

\section{References}

[1] A. Bellaachia and E. Guven. Predicting breast cancer survivability using data mining techniques. Scientific Data Mining Workshop, in Conjunction with the 2006 SIAM Conference on Data Mining, 2006.

[2] R. Bouckaert. Naive bayes classifiers that perform well with continuous variables. In AI 2004: Advances in Artificial Intelligence, volume 3339, pages 1089-1094. Springer Berlin / Heidelberg, 2005.
[3] D. Delen, G. Walker, and A. Kadam. Predicting breast cancer survivability: a comparison of three data mining methods. Artificial Intelligence in Medicine, 34(2):113-127, 2005.

[4] J. Garibaldi, D. Soria, F. Ambrogi, A. Green, D. Powe, E. Rakha, R. Macmillan, R. Blamey, G. Ball, P. Lisboa, T. Etchells, P. Boracchi, E. Biganzoli, and I. Ellis. Identification of key breast cancer phenotypes. Submitted to European Journal of Cancer, 2008.

[5] S. Haykin. Neural Networks: A Comprehensive Foundation. Prentice Hall, 2 edition, 1998.

[6] G. John and P. Langley. Estimating continuous distributions in bayesian classifiers. Proceeding of the Eleventh Conference on Uncertainty in Artificial Intelligence, 1995.

[7] T. Mitchell. Machine Learning. McGraw-Hill, 1997.

[8] J. Nahar, Y.-P. Chen, and S. Ali. Kernel-based naive bayes classifier for breast cancer prediction. Journal of Biological System, 15(1):17-25, 2007.

[9] J. Quinlan. C4.5: Programs for Machine Learning. Morgan Kaufmann, Los Altos, California, 1993.

[10] P. Royston. Algorithm as 181: The w test for normality. Applied Statistics, 31:176180, 1982.

[11] I. Witten and E. Frank. Data Mining: Practical Machine Learning Tools and Techniques with Java Implementations. Morgan Kaufmann, San Francisco, 2000. 